\title{
Conservation Laws of The Generalized Riemann Equations
}

Binfang Gao, Kai Tian, Q. P. Liu, Lujuan Feng

To cite this article: Binfang Gao, Kai Tian, Q. P. Liu, Lujuan Feng (2018) Conservation Laws of The Generalized Riemann Equations, Journal of Nonlinear Mathematical Physics 25:1, 122-135, DOI: https://doi.org/10.1080/14029251.2018.1440746

To link to this article: https://doi.org/10.1080/14029251.2018.1440746

Published online: 04 January 2021 


\title{
Conservation Laws of The Generalized Riemann Equations
}

\author{
Binfang Gao, Kai Tian, Q. P. Liu and Lujuan Feng \\ Department of Mathematics, China University of Mining and Technology \\ Beijing, 100083, People's Republic of China \\ tiankai@lsec.cc.ac.cn (K.Tian)
}

Received 7 June 2017

Accepted 23 October 2017

\begin{abstract}
Two special classes of conserved densities involving arbitrary smooth functions are explicitly formulated for the generalized Riemann equation at arbitrary $N$. The particular case when $N=2$ covers most of the known conserved densities of the equation, and the result is also extended to the famous Gurevich-Zybin, MongeAmpere and two-component Hunter-Saxton equations by considering certain reductions.

Keywords: conserved densities; generalized Riemann equation; Gurevich-Zybin equation; Monge-Ampere equation; reciprocal transformation.
\end{abstract}

2000 Mathematics Subject Classification: 35L65, 35L60, 37K05

\section{Introduction}

The Riemann equation

$$
u_{t}+u u_{x}=0
$$

where $u=u(x, t)$ and subscripts denote specified partial derivatives, also known as the inviscid Burgers equation or the Hopf equation in the literature, has been extensively studied as a prototype for many properties related to hyperbolic systems [16]. It is interesting that the Riemann equation (1.1) was generalized to

$$
\left(\partial_{t}+u \partial_{x}\right)^{N} u=0, \quad\left(N \in \mathbb{Z}_{+}\right)
$$

(see $[3,12,13]$ and the references there), which could be reformulated as an $N$-component system, namely

$$
u_{j, t}=\left(1-\delta_{j N}\right) u_{j+1}-u_{1} u_{j, x}, \quad(j=1,2, \cdots, N)
$$

where $u_{1}=u, u_{j+1}=\left(\partial_{t}+u \partial_{x}\right) u_{j}(1 \leq j<N)$ and $\delta_{j N}$ is the Kronecker delta. Eq. (1.2), or equivalently the system (1.3), is referred to as the generalized Riemann equation, and has been proven to be integrable in the sense of admitting a universal matrix Lax representation for arbitrary $N$ in Ref. [11]. Furthermore, the systems at $N=2,3$ and 4 have been shown to possess bi-Hamiltonian structures $[12,13]$. There are intimate relations among the generalized Riemann equations and some physically important models. In fact, the generalized Riemann equation at $N=2$ could be viewed as a regular Whitham type system [3], and reduced to the Gurevich-Zybin system [10] or an equation

${ }^{*}$ Corresponding author. 
describing non-local gas dynamics as well [2]. Through a certain transformation, an $N$-component extension of the Hunter-Saxton equation [6,7] was deduced from the generalized Riemann equation (1.3) in Ref. [11].

Many efforts have been made to construct conserved densities for the generalized Riemann equations (in general or special cases) [2,10-12]. It turns out that these equations have extraordinary features, namely abundant conserved densities of non-polynomial type, which are mainly generated by iterative procedures [11,12], such as $\left(F, G\right.$ and $H$ are smooth functions of $u_{1}, u_{2}, \cdots, u_{N}$ and their derivatives)

- If $F$ solves a linear equation $\partial_{t} F=k u_{1, x} F+u_{1} F_{x}$, then $F^{1 / k}$ is a conserved density such that [12]

$$
\partial_{t}\left(F^{1 / k}\right)=\partial_{x}\left(u_{1} F^{1 / k}\right) .
$$

- If there is a conservation law $\partial_{t} H=\lambda \partial_{x}\left(u_{1} H\right)$ and $F$ satisfies $\partial_{t} F=2 \lambda u_{1, x} F+\lambda u_{1} F_{x}$, then $F / H$ is a new conserved density such that [12]

$$
\partial_{t}\left(\frac{F}{H}\right)=\lambda \partial_{x}\left(u_{1} \frac{F}{H}\right) .
$$

- Let $R_{k}$ be a first order differential operator defined as

$$
R_{k}=u_{N, x}^{k-1} \partial_{x} u_{N, x}^{-k} \quad(k \neq 0)
$$

and $G$ and $F$ respectively satisfy

$$
\partial_{t} G=-k u_{1, x} G-u_{1} G_{x}, \quad \partial_{t} F=-(k-1) u_{1, x} F-u_{1} F_{x},
$$

then for non-negative integers $m$ and $n$ both $\left(R_{k}^{m} G\right)^{1 / k}$ and $\left(R_{k}^{m} G\right) /\left(R_{k-1}^{n} F\right)$ are conserved densities such that [11]

$$
\partial_{t}\left(\left(R_{k}^{m} G\right)^{1 / k}\right)=-\partial_{x}\left(u_{1}\left(R_{k}^{m} G\right)^{1 / k}\right), \quad \partial_{t}\left(\frac{R_{k}^{m} G}{R_{k-1}^{n} F}\right)=-\partial_{x}\left(u_{1} \frac{R_{k}^{m} G}{R_{k-1}^{n} F}\right) .
$$

Such diversity and richness of conserved densities distinguish the generalized Riemann equations from a typical integrable system such as the Korteweg-de Vries equation. Indeed, typical integrable systems normally possess infinitely many conserved densities of differential polynomial type, which may be generated by their linear spectral problems [1] or the Lenard scheme with bi-Hamiltonian structures [8].

It is interesting to observe that most conserved densities of the generalized Riemann equations in the literature surprisingly obey a common conservation law, namely

$$
\partial_{t}(F)=-\partial_{x}\left(u_{1} F\right),
$$

where the conserved density $F$ is a certain smooth function in $u_{1}, u_{2}, \cdots, u_{N}$ and their derivatives. This feature, which was also noticed by Popowicz [11], raises the following questions:

- Other than those listed above, are there new conserved densities which also fulfill Eq. (1.5)?

- Could the general solution of Eq. (1.5) be constructed under certain condition?

- Is it possible to find conservation laws of new types for the generalized Riemann equations? 
Indeed, available results for those systems, which are closely related to the generalized Riemann equations, motivate one to expect more general conserved densities for the latter. On one hand, the Riemann equation (1.1) was shown by Olver and Nutku to have a class of conservation laws [9], given by

$$
\partial_{t}\left(u_{x} G\left(z_{2}, z_{3}, \cdots, z_{n}\right)\right)=\partial_{x}\left(-u u_{x} G\left(z_{2}, z_{3}, \cdots, z_{n}\right)\right)
$$

where $z_{j}=\left(u_{x}^{-1} \partial_{x}\right)^{j-2}\left(u_{x}^{-3} u_{x x}\right)(j \geq 2)$, and $G$ is an arbitrary smooth function of its arguments. On the other hand, similar results have recently been achieved for the Hunter-Saxton equation [15], which also admits conserved densities involving arbitrary smooth functions.

The aim of this paper is to provide answers to above questions and offer better understanding on richness of conserved densities of the generalized Riemann equations. A method will be proposed to solve Eq. (1.5) so that conserved densities of the generalized Riemann equations are constructed. For the general $N$, a set of appropriate dynamical variables will be introduced and with the help of those variables the assumed conservation law will be brought to a first order linear Partial Differential Equation (PDE). Conserved densities will be obtained as the general solution to the linear PDE by the standard method of characteristics, and hence would involve arbitrary smooth functions which cast light on the diversity and richness of conserved densities presented by various authors $[2,10$ 12]. Furthermore, conservation laws, which are different from Eq. (1.5), will be directly constructed from a simple ansatz on their densities. The generalized Riemann equation at $N=2$, which appears to be the most important case and has close relation with several known systems, will be taken as an enlightening example to explain our methodology in detail, while results in the generic case are presented without proofs.

This paper is organized as follows. In section 2, the generalized Riemann equation at $N=2$ is shown to have two kinds of conserved densities involving arbitrary functions by solving some assumed conservation laws (like Eq. (1.5)) with suitable dynamical variables. Three well known systems related to it, namely the Gurevich-Zybin system, the Monge-Ampere equation and the twocomponent Hunter-Saxton equation, are considered in section 3, and their conserved densities are deduced from those of the generalized Riemann equation at $N=2$. In section 4 , new dynamical variables are introduced for the generalized Riemann equation at arbitrary $N$, and they enable us to explicitly figure out conserved densities involving arbitrary smooth functions. As illustrations, general formulas are applied to the cases when $N=3,4$. Conclusions are given in the last section.

\section{The $N=2$ case}

By rescaling dependent variables as $u_{1}=-2 u$ and $u_{2}=4 v$, the generalized Riemann equation at $N=2$ is rewritten as

$$
\left\{\begin{array}{l}
u_{t}=2 u u_{x}-2 v, \\
v_{t}=2 u v_{x} .
\end{array}\right.
$$

In this section, suitable changes of variables are introduced to linearize the $x$-derivative of the system (2.1) and convert an assumed conservation law of the system (2.1), i.e.

$$
\partial_{t}\left(F\left(u_{x}, v_{x}, u_{2 x}, v_{2 x}, \cdots, u_{n x}, v_{n x}\right)\right)=\partial_{x}\left(2 u F\left(u_{x}, v_{x}, u_{2 x}, v_{2 x}, \cdots, u_{n x}, v_{n x}\right)\right),
$$


to a first order PDE, where $u_{j x}=\left(\partial_{x}^{j} u\right), v_{j x}=\left(\partial_{x}^{j} v\right)(j \geq 2)$. Solving this PDE by quadrature and inversely changing its general solutions to original variables will give us all conserved densities such that Eq. (2.2) is solved. Guided by this partial result, we iteratively define a set of dynamical variables through differential relations, and determine their evolutions when the system (2.1) holds. With the help of these dynamical variables, we solve all local conserved densities, which may explicitly depend on $u$ and $v$, from the assumed conservation law of the system (2.1). Also, a method is presented to construct conservation laws of new type from arbitrary smooth function in these dynamical variables.

Differentiating the system (2.1) with respect to $x$ yields

$$
\begin{aligned}
& u_{x t}=2 u u_{x x}+2 u_{x}^{2}-2 v_{x}, \\
& v_{x t}=\partial_{x}\left(2 u v_{x}\right) .
\end{aligned}
$$

The trivial conservation law (2.3b) allows us to introduce the following reciprocal transformation

$$
\mathscr{T}_{1}:(x, t, u, v) \mapsto(y, \tau, \bar{u}, \bar{v}),
$$

where new variables are defined by

$$
\mathrm{d} y=v_{x} \mathrm{~d} x+\left(2 u v_{x}\right) \mathrm{d} t, \quad \mathrm{~d} \tau=\mathrm{d} t, \quad \bar{u}(y, \tau)=u_{x}, \quad \bar{v}(y, \tau)=v_{x} .
$$

It is easy to see that the partial derivatives with respect to $x$ or $t$ transform according to

$$
\partial_{x}=\bar{v} \partial_{y}, \quad \partial_{t}=2 u \bar{v} \partial_{y}+\partial_{\tau} .
$$

Applying $\mathscr{T}_{1}$ to Eqs. (2.3a) and (2.3b) respectively, we have

$$
\begin{aligned}
& 0=u_{x t}-\left(2 u u_{x x}+2 u_{x}^{2}-2 v_{x}\right)=\bar{u}_{t}-\left(2 u \bar{u}_{x}+2 \bar{u}^{2}-2 \bar{v}\right)=2 u \bar{v} \bar{u}_{y}+\bar{u}_{\tau}-\left(2 u \bar{v} \bar{u}_{y}+2 \bar{u}^{2}-2 \bar{v}\right), \\
& 0=v_{x t}-\partial_{x}\left(2 u v_{x}\right)=\bar{v}_{t}-\bar{v} \partial_{y}(2 u \bar{v})=2 u \bar{v} \bar{v}_{y}+\bar{v}_{\tau}-\left(2 u \bar{v} \bar{v}_{y}+2 \bar{u} \bar{v}\right) .
\end{aligned}
$$

Hence, Eqs. (2.3a) and (2.3b) are converted to

$$
\left\{\begin{array}{l}
\bar{u}_{\tau}=2 \bar{u}^{2}-2 \bar{v}, \\
\bar{v}_{\tau}=2 \bar{u} \bar{v} .
\end{array}\right.
$$

Now we introduce the following new dependent variables via

$$
\mathscr{T}_{2}:(\bar{u}, \bar{v}) \mapsto(p, q)=\left(\frac{\bar{u}^{2}-2 \bar{v}}{\bar{v}^{2}}, \frac{\bar{u}}{\bar{v}}\right),
$$

then the system (2.5) is linearized to

$$
\left\{\begin{array}{l}
p_{\tau}=0 \\
q_{\tau}=-2
\end{array}\right.
$$

With the aid of $\mathscr{T}_{1}$ and $\mathscr{T}_{2}$, one can reduce the assumed conservation law (2.2) of the system (2.1) to a solvable PDE, and figure out conserved densities. According to (2.4), Eq. (2.2) is changed 
by $\mathscr{T}_{1}$ to (We temporarily omit the arguments of $F$ for simplicity.)

$$
\begin{aligned}
0 & =\partial_{t} F-2 u_{x} F-2 u \partial_{x} F \\
& =2 u \bar{v} \partial_{y} \mathscr{T}_{1}(F)+\partial_{\tau} \mathscr{T}_{1}(F)-2 \bar{u} \mathscr{T}_{1}(F)-2 u \bar{v} \partial_{y} \mathscr{T}_{1}(F) \\
& =\partial_{\tau} \mathscr{T}_{1}(F)-2 \bar{u} \mathscr{T}_{1}(F) .
\end{aligned}
$$

By means of $\mathscr{T}_{2}$ or in terms of $p$ and $q$, above equation becomes

$$
\partial_{\tau}\left(\widehat{F}\left(p, q, p_{y}, q_{y}, \cdots, p_{(n-1) y}, q_{(n-1) y}\right)\right)=\frac{4 q}{q^{2}-p} \widehat{F}\left(p, q, p_{y}, q_{y}, \cdots, p_{(n-1) y}, q_{(n-1) y}\right),
$$

where

$$
\widehat{F}\left(p, q, p_{y}, q_{y}, \cdots, p_{(n-1) y}, q_{(n-1) y}\right)=\left(\mathscr{T}_{2} \circ \mathscr{T}_{1}\right)\left(F\left(u_{x}, v_{x}, u_{2 x}, v_{2 x}, \cdots, u_{n x}, v_{n x}\right)\right) .
$$

As Eq. (2.2) is assumed to be a conservation law, it holds on the solutions to the system (2.1). Equivalently, Eq. (2.7) holds provided that $p$ and $q$ solve the system (2.6). It is now easy to show that, on solutions to the system (2.6), Eq. (2.7) reduces to

$$
\frac{\partial \widehat{F}}{\partial q}+\frac{2 q}{q^{2}-p} \widehat{F}=0
$$

which can be solved by quadratures, and the general solution is

$$
\widehat{F}=\frac{1}{q^{2}-p} G\left(p, p_{y}, q_{y}, \cdots, p_{(n-1) y}, q_{(n-1) y}\right),
$$

where $G$ is an arbitrary smooth function of its arguments. Reformulating this solution in terms of $u$, $v$ and their derivatives gives us the explicit conserved densities of the system (2.1).

Based on the discussions so far, we conclude that Eq. (2.2) is a conservation law of the system (2.1) if and only if the density $F\left(u_{x}, v_{x}, u_{2 x}, v_{2 x}, \cdots, u_{n x}, v_{n x}\right)$ is of the form

$$
\frac{v_{x}}{2} G\left(\alpha^{(1)}, \alpha^{(2)}, \beta^{(2)}, \cdots, \alpha^{(n)}, \beta^{(n)}\right),
$$

where $G$ is an arbitrary smooth function, and its arguments are defined as

$$
\alpha^{(j)}=\left(v_{x}^{-1} \partial_{x}\right)^{j-1}\left(u_{x}^{2} v_{x}^{-2}-2 v_{x}^{-1}\right), \quad \beta^{(j)}=\left(v_{x}^{-1} \partial_{x}\right)^{j-1}\left(u_{x} v_{x}^{-1}\right), \quad\left(j \in \mathbb{Z}_{+}\right) .
$$

It is remarked that, the quantities $\alpha^{(j)}$ 's and $\beta^{(j)}$ 's, which are simply deduced by applying $\left(\mathscr{T}_{1}^{-1} \circ \mathscr{T}_{2}^{-1}\right)$ to $p, q$ and their derivatives with respect to $y$, play the important roles in formulating conserved densities. Next, we demonstrate that they have interesting properties, which lead us to more general results and bring us new ideas to study conservation laws in the general case as well.

Lemma 2.1. When the system (2.1) holds,

$$
\partial_{t} \alpha^{(j)}=2 u\left(\partial_{x} \alpha^{(j)}\right) \quad \text { and } \quad \partial_{t} \beta^{(j)}=2 u\left(\partial_{x} \beta^{(j)}\right)-2 \delta_{j, 1}, \quad\left(j \in \mathbb{Z}_{+}\right) .
$$


Proof. For $\alpha^{(1)}=u_{x}^{2} v_{x}^{-2}-2 v_{x}^{-1}$ and $\beta^{(1)}=u_{x} v_{x}^{-1}$, direct calculations give us

$$
\partial_{t} \alpha^{(1)}=2 u\left(\partial_{x} \alpha^{(1)}\right) \quad \text { and } \quad \partial_{t} \beta^{(1)}=2 u\left(\partial_{x} \beta^{(1)}\right)-2 .
$$

It is noticed that both $\alpha^{(j)}$ and $\beta^{(j)}(j \geq 2)$ are defined by the same recursive relation

$$
\gamma^{(j+1)}=\left(v_{x}^{-1} \partial_{x}\right) \gamma^{(j)}
$$

Let us assume $\partial_{t} \gamma^{(j)}=2 u\left(\partial_{x} \gamma^{(j)}\right)+c_{j}$, where $c_{j}$ stands for certain constant, then calculating time evolution of both sides of the recursive relation (2.11), we obtain

$\partial_{t} \gamma^{(j+1)}=-v_{x}^{-2} v_{x t}\left(\partial_{x} \gamma^{(j)}\right)+v_{x}^{-1}\left(\partial_{x} \partial_{t} \gamma^{(j)}\right)=2 u\left(-v_{x}^{-2} v_{2 x}\left(\partial_{x} \gamma^{(j)}\right)+v_{x}^{-1}\left(\partial_{x}^{2} \gamma^{(j)}\right)\right)=2 u\left(\partial_{x} \gamma^{(j+1)}\right)$

Hence, the conclusion holds for all $\alpha^{(j)}$ and $\beta^{(j)}$.

Lemma 2.2. For any $n \in \mathbb{Z}_{+}$, the change of dynamical variables

$$
\Gamma:\left(u_{x}, v_{x}, u_{2 x}, v_{2 x}, \cdots, u_{n x}, v_{n x}\right) \mapsto\left(\alpha^{(1)}, \beta^{(1)}, \alpha^{(2)}, \beta^{(2)}, \cdots, \alpha^{(n)}, \beta^{(n)}\right)
$$

is invertible.

Proof. To prove the invertibility of $\Gamma$, it would be enough to formulate $\left(u_{x}, v_{x}, \cdots, u_{n x}, v_{n x}\right)$ in terms of $\left(\alpha^{(1)}, \beta^{(1)}, \alpha^{(2)}, \beta^{(2)}, \cdots, \alpha^{(n)}, \beta^{(n)}\right)$. Solving $u_{x}$ and $v_{x}$ from explicit expressions of $\alpha^{(1)}$ and $\beta^{(1)}$ (cf. Eqs. (2.10)) yields

$$
u_{x}=\frac{2 \beta^{(1)}}{\left(\beta^{(1)}\right)^{2}-\alpha^{(1)}}, \quad v_{x}=\frac{2}{\left(\beta^{(1)}\right)^{2}-\alpha^{(1)}} .
$$

Inferred from Eqs. (2.10), we get

$$
\left(\begin{array}{l}
\alpha^{(j)} \\
\beta^{(j)}
\end{array}\right)=\left(\begin{array}{cc}
2 u_{x} v_{x}^{-j-1} & 2\left(v_{x}-u_{x}^{2}\right) v_{x}^{-j-2} \\
v_{x}^{-j} & -u_{x} v_{x}^{-j-1}
\end{array}\right)\left(\begin{array}{l}
u_{j x} \\
v_{j x}
\end{array}\right)+\mathbb{G}_{1}\left(u_{x}, v_{x}, \cdots, u_{(j-1) x}, v_{(j-1) x}\right),
$$

where $\mathbb{G}_{1}$ is a 2 -dimensional vector function. The $2 \times 2$ matrix in the right hand side is non-singular, so we could solve $u_{j x}$ and $v_{j x}$ step by step, and formulate them in terms of $\left(\alpha^{(1)}, \beta^{(1)}, \alpha^{(2)}, \beta^{(2)}, \cdots, \alpha^{(j)}, \beta^{(j)}\right)$.

Via the invertible transformation $\Gamma$, any smooth function $F\left(u, v, u_{x}, v_{x}, \cdots, u_{n x}, v_{n x}\right)$ could be reformulated as $\widehat{F}\left(u, v, \alpha^{(1)}, \beta^{(1)}, \cdots, \alpha^{(n)}, \beta^{(n)}\right)=\Gamma\left(F\left(u, v, u_{x}, v_{x}, \cdots, u_{n x}, v_{n x}\right)\right)$ and vice versa, then its time-evolution is easily determined according to Lemma 2.1. Taking account of both lemmas, we will determine all conserved densities, which may explicitly depend on $u$ and $v$, by solving the assumed conservation law

$$
\partial_{t}\left(F\left(u, v, u_{x}, v_{x}, \cdots, u_{n x}, v_{n x}\right)\right)=\partial_{x}\left(2 u F\left(u, v, u_{x}, v_{x}, \cdots, u_{n x}, v_{n x}\right)\right),
$$

in coordinates $\left(u, v, \alpha^{(1)}, \beta^{(1)}, \cdots, \alpha^{(n)}, \beta^{(n)}\right)$. 
Indeed, in terms of $\alpha^{(j)}$ 's and $\beta^{(j)}$ 's, (2.12) appears as

$$
\partial_{t}\left(\widehat{F}\left(u, v, \alpha^{(1)}, \beta^{(1)}, \cdots, \alpha^{(n)}, \beta^{(n)}\right)\right)-\partial_{x}\left(2 u \widehat{F}\left(u, v, \alpha^{(1)}, \beta^{(1)}, \cdots, \alpha^{(n)}, \beta^{(n)}\right)\right)=0,
$$

which, after expanding all derivatives, yields

$$
\begin{aligned}
\frac{\partial \widehat{F}}{\partial u} u_{t}+\frac{\partial \widehat{F}}{\partial v} v_{t} & +\sum_{j=0}^{n} \frac{\partial \widehat{F}}{\partial \alpha^{(j)}}\left(\partial_{t} \alpha^{(j)}\right)+\sum_{j=0}^{n} \frac{\partial \widehat{F}}{\partial \beta^{(j)}}\left(\partial_{t} \beta^{(j)}\right)-2 u_{x} \widehat{F} \\
& -2 u\left(\frac{\partial \widehat{F}}{\partial u} u_{x}+\frac{\partial \widehat{F}}{\partial v} v_{x}+\sum_{j=0}^{n} \frac{\partial \widehat{F}}{\partial \alpha^{(j)}}\left(\partial_{x} \alpha^{(j)}\right)+\sum_{j=0}^{n} \frac{\partial \widehat{F}}{\partial \beta^{(j)}}\left(\partial_{x} \beta^{(j)}\right)\right)=0
\end{aligned}
$$

Now taking into consideration the system (2.1) and Lemma 2.1, we find that above equation is simplified as

$$
v \frac{\partial \widehat{F}}{\partial u}+\frac{\partial \widehat{F}}{\partial \beta^{(1)}}+\frac{2 \beta^{(1)}}{\left(\beta^{(1)}\right)^{2}-\alpha^{(1)}} \widehat{F}=0
$$

which is a first-order non-homogeneous linear PDE for $\widehat{F}$. Solving it by the standard method of characteristics, we obtain

$$
\widehat{F}=\frac{v_{x}}{2} G\left(v, \alpha^{(1)}, \beta^{(1)}-u v^{-1}, \alpha^{(2)}, \beta^{(2)}, \cdots, \alpha^{(n)}, \beta^{(n)}\right),
$$

where $G$ is an arbitrary smooth function of its arguments. Taking account of Eqs. (2.10) we find, in terms of the original variables, the conserved density namely $\Gamma^{-1}(\widehat{F})$. The result is summarized as

Proposition 2.1. The system (2.1) admits the conservation law (2.12) if and only if

$$
F\left(u, v, u_{x}, v_{x}, \cdots, u_{n x}, v_{n x}\right)=\frac{v_{x}}{2} G\left(v, \alpha^{(1)}, \beta^{(1)}-u v^{-1}, \alpha^{(2)}, \beta^{(2)}, \cdots, \alpha^{(n)}, \beta^{(n)}\right),
$$

where $\alpha^{(j)}$ and $\beta^{(j)}$ are defined by Eqs. (2.10), and $G$ is an arbitrary smooth function of its arguments.

Remark 2.1. In particular, if the conserved densities of the type (2.13) do not explicitly depend on $u$ and $v$, we recover the conserved densities of the type (2.9).

In the rest part of the section, we manage to find conservation laws other than in the form of Eq. (2.2) for the system (2.1). Since the conserved densities of the type (2.9) do not explicitly depend on $\beta^{(1)}$, it is interesting to construct the new conservation laws explicitly depending on $\beta^{(1)}$. Let us consider a smooth function

$$
H\left(u_{x}, v_{x}, \cdots, u_{n x}, v_{n x}\right)=v_{x} P\left(\alpha^{(1)}, \beta^{(1)}, \alpha^{(2)}, \beta^{(2)}, \cdots, \alpha^{(n)}, \beta^{(n)}\right) .
$$

On solutions to the system (2.1), by means of Lemma 2.1 we straightforwardly get

$$
\partial_{t} H=\partial_{x}\left(2 u v_{x} P\right)-2 v_{x} \frac{\partial P}{\partial \beta^{(1)}} .
$$

A simple but interesting observation on the above expression is that if there exists a function $Q\left(\alpha^{(1)}, \beta^{(1)}, \alpha^{(2)}, \beta^{(2)}, \cdots, \alpha^{(n-1)}, \beta^{(n-1)}\right)$ such that

$$
\partial_{x} Q=-2 v_{x} \frac{\partial P}{\partial \beta^{(1)}},
$$


then $H=v_{x} P\left(\alpha^{(1)}, \beta^{(1)}, \alpha^{(2)}, \beta^{(2)}, \cdots, \alpha^{(n)}, \beta^{(n)}\right)$ would be a conserved density such that $\partial_{t} H=$ $\partial_{x}(2 u H+Q)$. Eq. (2.14), which may be used to determine $P$ for a given function $Q$, is written as

$$
\frac{\partial P}{\partial \beta^{(1)}}=-\frac{1}{2} \sum_{j=1}^{n-1}\left(\frac{\partial Q}{\partial \alpha^{(j)}} \alpha^{(j+1)}+\frac{\partial Q}{\partial \beta^{(j)}} \beta^{(j+1)}\right),
$$

since

$$
\partial_{x} Q=\sum_{j=1}^{n-1}\left(\frac{\partial Q}{\partial \alpha^{(j)}}\left(\partial_{x} \alpha^{(j)}\right)+\frac{\partial Q}{\partial \beta^{(j)}}\left(\partial_{x} \beta^{(j)}\right)\right)=v_{x} \sum_{j=1}^{n-1}\left(\frac{\partial Q}{\partial \alpha^{(j)}} \alpha^{(j+1)}+\frac{\partial Q}{\partial \beta^{(j)}} \beta^{(j+1)}\right),
$$

which is implied by definitions of $\alpha^{(j)}$ 's and $\beta^{(j)}$ 's, i.e. Eqs. (2.10).

We summarize above discussions as

Proposition 2.2. When the system (2.1) holds, given an arbitrary smooth function $Q\left(\alpha^{(1)}, \beta^{(1)}, \alpha^{(2)}, \beta^{(2)}, \cdots, \alpha^{(n-1)}, \beta^{(n-1)}\right)$, let

$$
P=-\frac{1}{2} \int \sum_{j=1}^{n-1}\left(\frac{\partial Q}{\partial \alpha^{(j)}} \alpha^{(j+1)}+\frac{\partial Q}{\partial \beta^{(j)}} \beta^{(j+1)}\right) \mathrm{d} \beta^{(1)},
$$

then $H=v_{x} P$ (after replacing all $\alpha^{(j)}$ and $\beta^{(j)}$ by (2.10)) is conserved and satisfies the conservation law $\partial_{t} H=\partial_{x}(2 u H+Q)$.

As an implementation of Proposition 2.2, we construct a conserved density from the smooth function $Q\left(\alpha^{(1)}\right) \beta^{(1)}$. Now the formula (2.15) leads to

$$
\begin{aligned}
P & =-\frac{1}{2} \int\left(Q^{\prime}\left(\alpha^{(1)}\right) \alpha^{(2)} \beta^{(1)}+Q\left(\alpha^{(1)}\right) \beta^{(2)}\right) \mathrm{d} \beta^{(1)} \\
& =-\frac{1}{4} Q^{\prime}\left(\alpha^{(1)}\right) \alpha^{(2)}\left(\beta^{(1)}\right)^{2}-\frac{1}{2} Q\left(\alpha^{(1)}\right) \beta^{(1)} \beta^{(2)}+T\left(\alpha^{(1)}, \alpha^{(2)}, \beta^{(2)}\right),
\end{aligned}
$$

where $Q^{\prime}\left(\alpha^{(1)}\right)=\partial Q / \partial \alpha^{(1)}$ and $T\left(\alpha^{(1)}, \alpha^{(2)}, \beta^{(2)}\right)$ is the "constant" of integration. Then, we obtain a conserved density

$$
H\left(u_{x}, v_{x}, u_{x x}, v_{x x}\right)=-\frac{1}{4} v_{x} Q^{\prime}\left(\alpha^{(1)}\right) \alpha^{(2)}\left(\beta^{(1)}\right)^{2}-\frac{1}{2} v_{x} Q\left(\alpha^{(1)}\right) \beta^{(1)} \beta^{(2)}+v_{x} T\left(\alpha^{(1)}, \alpha^{(2)}, \beta^{(2)}\right)
$$

such that

$$
\partial_{t} H\left(u_{x}, v_{x}, u_{x x}, v_{x x}\right)=\partial_{x}\left(2 u H\left(u_{x}, v_{x}, u_{x x}, v_{x x}\right)+Q\left(\alpha^{(1)}\right) \beta^{(1)}\right) .
$$

It is noticed that $v_{x} T\left(\alpha^{(1)}, \alpha^{(2)}, \beta^{(2)}\right)$ is also a conserved density as implied by Proposition 2.1 , and satisfies

$$
\partial_{t}\left(v_{x} T\left(\alpha^{(1)}, \alpha^{(2)}, \beta^{(2)}\right)\right)=\partial_{x}\left(2 u v_{x} T\left(\alpha^{(1)}, \alpha^{(2)}, \beta^{(2)}\right)\right) .
$$

So the conserved density $H\left(u_{x}, v_{x}, u_{x x}, v_{x x}\right)$ may be replaced by

$$
-\frac{1}{4} v_{x} Q^{\prime}\left(\alpha^{(1)}\right) \alpha^{(2)}\left(\beta^{(1)}\right)^{2}-\frac{1}{2} v_{x} Q\left(\alpha^{(1)}\right) \beta^{(1)} \beta^{(2)},
$$

and in this way the conservation law (2.16) could be simplified. 
To conclude this section, we point out that while above results show that the generalized Riemann equation at $N=2$ does possess abundant conserved densities, they by no means exhaust all possibilities since there are extra conservation laws explicitly depending on time [11], such as

$$
\begin{aligned}
\partial_{t}\left(2 t u_{x} v+v\right) & =\partial_{x}\left(t\left(4 u u_{x} v-2 v^{2}\right)+2 u v\right) \\
\partial_{t}\left(u+2 t v+2 t^{2} u_{x} v\right) & =\partial_{x}\left(t^{2}\left(4 u u_{x} v-2 v^{2}\right)+4 t u v+u^{2}\right) .
\end{aligned}
$$

\section{The systems related to the $N=2$ case}

The generalized Riemann equation at $N=2$ is interesting since it is associated with some important systems. In fact, through the appropriate changes of variables and/or reductions, the system (2.1) is connected/reduced to the Gurevich-Zybin, Monge-Ampere, two-component Hunter-Saxton and Hunter-Saxton equations. In this section, we demonstrate that the results obtained in last section allow us to construct the conserved densities for those systems, and we concentrate on the reductions of the conserved densities (2.13) in particular.

\subsection{The Gurevich-Zybin system and the Monge-Ampere equation}

For the system (2.1), let $u=-\hat{u} / 2$ and $v=-\Phi_{x} / 4$, then the first equation is changed to

$$
\hat{u}_{t}+\hat{u} \hat{u}_{x}+\Phi_{x}=0
$$

while the second equation to

$$
\Phi_{x t}+\hat{u} \Phi_{x x}=0 .
$$

Differentiating Eq. (3.2) with respect to $x$ and letting $\rho=\Phi_{x x}$, we obtain

$$
\partial_{t}(\rho)+\partial_{x}(\hat{u} \rho)=0 .
$$

The coupled system (3.1) and (3.3) is nothing but the Gurevich-Zybin system in the one-dimensional case $[4,5]$, which has been shown to be linearisable and possesses infinitely many local Hamiltonian structures, local Lagrangian representations and local conservation laws (symmetries) [10]. As observed by Pavlov, solving $\hat{u}$ from Eq. (3.2) and substituting it into a conservation law of the Gurevich-Zybin system, i.e.

$$
\partial_{t}(\rho \hat{u})+\partial_{x}\left(\rho \hat{u}^{2}+\Phi_{x}^{2} / 2\right)=0
$$

we obtain the Monge-Ampere equation

$$
\Phi_{t t}=\frac{\Phi_{x t}^{2}}{\Phi_{x x}}+\frac{1}{2} \Phi_{x}^{2}
$$

Taking above relations into consideration, one could deduce their conserved densities from those of the system (2.1). For instance, according to Proposition 2.1, the Gurevich-Zybin system (3.1) and 
(3.3) has the conserved densities of the form

$$
\rho G\left(\left(\partial_{x}^{-1} \rho\right), \bar{\alpha}^{(1)}, \bar{\beta}^{(1)}-\hat{u}\left(\partial_{x}^{-1} \rho\right)^{-1}, \bar{\alpha}^{(2)}, \bar{\beta}^{(2)}, \cdots, \bar{\alpha}^{(n)}, \bar{\beta}^{(n)}\right)
$$

such that $\partial_{t}(\rho G)=\partial_{x}(-\hat{u} \rho G)$, where $\partial_{x}^{-1}$ denotes the inverse of $\partial_{x}$ such that $\partial_{x}^{-1} \partial_{x}=\partial_{x} \partial_{x}^{-1}=1$, while

$$
\bar{\alpha}^{(j)}=\left(\rho^{-1} \partial_{x}\right)^{j-1}\left(\left(\hat{u}_{x}^{2}+2 \rho\right) \rho^{-2}\right) \quad \text { and } \quad \bar{\beta}^{(j)}=\left(\rho^{-1} \partial_{x}\right)^{j-1}\left(\hat{u}_{x} \rho^{-1}\right), \quad\left(j \in \mathbb{Z}_{+}\right) .
$$

Similarly, replacing $u$ by $\Phi_{x t} /\left(2 \Phi_{x x}\right)$, while $v$ by $-\Phi_{x} / 4$ in (2.13) yields the conserved density of the Monge-Ampere equation (3.4).

\subsection{The two-component Hunter-Saxton equation}

Let $v_{x}=\left(u_{x}^{2}+\eta^{2}\right) / 2$, then the system (2.3a)-(2.3b) is converted to

$$
\left\{\begin{aligned}
u_{x t} & =2 u u_{x x}+u_{x}^{2}-\eta^{2} \\
\eta_{t} & =2(u \eta)_{x}
\end{aligned}\right.
$$

which reduces to the Hunter-Saxton equation $[6,7]$ when $\eta=0$, and is referred to as the twocomponent Hunter-Saxton equation [10,12].

Replacing $v_{x}$ by $\left(u_{x}^{2}+\eta^{2}\right) / 2$ in (2.13) immediately gives us the conserved density

$$
\left(u_{x}^{2}+\eta^{2}\right) G\left(\partial_{x}^{-1}\left(u_{x}^{2}+\eta^{2}\right), \tilde{\alpha}^{(1)}, \tilde{\beta}^{(1)}-\frac{u}{\partial_{x}^{-1}\left(u_{x}^{2}+\eta^{2}\right)}, \tilde{\alpha}^{(2)}, \tilde{\beta}^{(2)}, \cdots, \tilde{\alpha}^{(n)}, \tilde{\beta}^{(n)}\right)
$$

for the two-component Hunter-Saxton equation (3.5), where

$$
\tilde{\alpha}^{(j)}=\left(\frac{\partial_{x}}{u_{x}^{2}+\eta^{2}}\right)^{j-1}\left(\frac{\eta^{2}}{\left(u_{x}^{2}+\eta^{2}\right)^{2}}\right) \quad \text { and } \quad \tilde{\beta}^{(j)}=\left(\frac{\partial_{x}}{u_{x}^{2}+\eta^{2}}\right)^{j-1}\left(\frac{u_{x}}{u_{x}^{2}+\eta^{2}}\right),\left(j \in \mathbb{Z}_{+}\right)
$$

and the corresponding conservation law is given by

$$
\partial_{t}\left(\left(u_{x}^{2}+\eta^{2}\right) G\right)=\partial_{x}\left(2 u\left(u_{x}^{2}+\eta^{2}\right) G\right) .
$$

When $\eta=0$, all $\tilde{\alpha}^{(j)}$ 's vanish and the density (3.6) reduces to

$$
u_{x}^{2} G_{2}\left(\left(\partial_{x}^{-1} u_{x}^{2}\right), u_{x}^{-1}-u\left(\partial_{x}^{-1} u_{x}^{2}\right)^{-1},\left(u_{x}^{-2} \partial_{x}\right) u_{x}^{-1},\left(u_{x}^{-2} \partial_{x}\right)^{2} u_{x}^{-1}, \cdots,\left(u_{x}^{-2} \partial_{x}\right)^{n-1} u_{x}^{-1}\right),
$$

which is nothing but the conserved density with arbitrary function of the Hunter-Saxton equation recently reported by two of the authors [15].

\section{The general case}

In this section, we extend the ideas proposed in section 2 to the general case. We shall introduce a set of dynamical variables for the generalized Riemann equation at arbitrary $N$, i.e. the system (1.3). With the help of these variables, we shall explicitly present conserved densities involving arbitrary smooth functions for the general case. As the validity of these results can be checked by direct calculations, we omit the details in the subsequent discussions. 
Let $Q_{0}=1 / u_{N, x}$ and $Q_{i}=u_{i, x} / u_{N, x}(i=1,2, \cdots, N)$, then it is shown by direct calculations that any monomial of degree $m$ in $Q_{i}$ 's, like $\prod_{k=1}^{m} Q_{i_{k}}\left(i_{k} \in\{0,1,2, \cdots, N\}\right)$ satisfies

$$
\partial_{t}\left(\prod_{k=1}^{m} Q_{i_{k}}\right)=-u_{1} \partial_{x}\left(\prod_{k=1}^{m} Q_{i_{k}}\right)+\left(\prod_{k=1}^{m} Q_{i_{k}}\right)\left(\sum_{k=1}^{m} \frac{Q_{i_{k}+1}}{Q_{i_{k}}}\right),
$$

when the system (1.3) holds. (As a convention, $Q_{N+1}$ in the above equation is always set to be 0 .) In order to construct conserved densities, we consider the fundamental dynamical variables which are defined as polynomials in $Q_{i}$ 's, i.e. $\alpha_{1}^{(1)}=Q_{N-1}$ and for $k=2,3, \cdots, N$

$$
\alpha_{k}^{(1)}=\left\{\begin{array}{cc}
Q_{N-l}^{2}+2 \sum_{i=0}^{l-1}(-1)^{l-i} Q_{N-i} Q_{N-k+i}, & (\text { if } k=2 l) \\
(-1)^{l+1} k\left(Q_{N-k}-Q_{N-1} Q_{N+1-k}\right)+\sum_{i=2}^{l}(-1)^{l+1-i}(k-2 i) Q_{N-i} Q_{N-k+i} & \\
+Q_{N-1}\left(Q_{N-l}^{2}+2 \sum_{i=1}^{l-1}(-1)^{l+i} Q_{N-i} Q_{N+1-k+i}\right) . & (\text { if } k=2 l+1)
\end{array}\right.
$$

Then, taking account of Eq. (4.1) we have

$$
\partial_{t} \alpha_{1}^{(1)}=-u_{1}\left(\partial_{x} \alpha_{1}^{(1)}\right)+1 \quad \text { and } \quad \partial_{t} \alpha_{k}^{(1)}=-u_{1}\left(\partial_{x} \alpha_{k}^{(1)}\right)(k=2,3, \cdots, N) .
$$

Remark 4.1. The introduction of those fundamental variables is motivated by the considerations of the $N=2$ case. Indeed, the dynamical variables $\alpha^{(1)}$ and $\beta^{(1)}$ defined by (2.10) for the $N=2$ case are $\alpha^{(1)}=4 \alpha_{2}^{(1)}$ and $\beta^{(1)}=-2 \alpha_{1}^{(1)}$ up to the scaling $u_{1}=-2 u, u_{2}=4 v$.

Other dynamical variables are iteratively defined as

$$
\alpha_{k}^{(j+1)}=\left(u_{N, x}^{-1} \partial_{x}\right) \alpha_{k}^{(j)} \quad(j \geq 1, k=1,2, \cdots, N) .
$$

On solutions to the system (1.3), a useful lemma is that suppose $\partial_{t} F=-u_{1}\left(\partial_{x} F\right)+b$, where $b$ is $a$ constant, then $G=\left(u_{N . x}^{-1} \partial_{x}\right) F$ is a quantity such that $\partial_{t} G=-u_{1}\left(\partial_{x} G\right)$. According to this property, we easily obtain

$$
\partial_{t} \alpha_{k}^{(j)}=-u_{1}\left(\partial_{x} \alpha_{k}^{(j)}\right) \quad(j \geq 2, k=1,2, \cdots, N) .
$$

Remark 4.2. The first order differential operator $u_{N, x}^{-1} \partial_{x}$ in iterative relations (4.3) of dynamical variables is different from the operator (1.4), which is used to generate non-polynomial conserved densities [11].

On the basis of Eqs. (4.2) and (4.4), it is straightforward to show that the system (1.3), namely the generalized Riemann equation at arbitrary $N$, has the following conservation laws

- $\partial_{t}(F)=\partial_{x}\left(-u_{1} F\right)$, where $F=u_{N, x} G\left(\alpha_{2}^{(1)}, \cdots, \alpha_{N}^{(1)}, \alpha_{1}^{(2)}, \cdots, \alpha_{N}^{(2)}, \cdots, \alpha_{1}^{(n)}, \cdots, \alpha_{N}^{(n)}\right)$, and $G$ is an arbitrary smooth function of its arguments.

- $\partial_{t}(H)=\partial_{x}\left(-u_{1} H+P\right)$, where $P\left(\alpha_{1}^{(1)}, \cdots, \alpha_{N}^{(1)}, \alpha_{1}^{(2)}, \cdots, \alpha_{N}^{(2)}, \cdots, \alpha_{1}^{(n-1)}, \cdots, \alpha_{N}^{(n-1)}\right)$ stands for an arbitrary smooth function of its arguments, and

$$
H=u_{N, x} \int\left(\sum_{i=1}^{N} \sum_{j=1}^{n-1} \frac{\partial P}{\partial \alpha_{i}^{(j)}} \alpha_{i}^{(j+1)}\right) \mathrm{d} \alpha_{1}^{(1)} .
$$


As implementations, the conserved densities in fundamental dynamical variables are presented for the generalized Riemann equations at $N=3,4$, and the corresponding conservation laws could be checked by direct calculations.

Example 4.1. When $N=3$, the fundamental dynamical variables are

$$
\alpha_{1}^{(1)}=Q_{2}, \quad \alpha_{2}^{(1)}=Q_{2}^{2}-2 Q_{3} Q_{1}, \quad \alpha_{3}^{(1)}=Q_{2}^{3}-3 Q_{1} Q_{2}+3 Q_{0} .
$$

Substituting $Q_{0}=1 / u_{3, x}$ and $Q_{i}=u_{i, x} / u_{3, x}(i=1,2,3)$ into them, we get

$$
\alpha_{1}^{(1)}=\frac{u_{2, x}}{u_{3, x}}, \quad \alpha_{2}^{(1)}=\frac{u_{2, x}^{2}-2 u_{1, x} u_{3, x}}{u_{3, x}^{2}}, \quad \alpha_{3}^{(1)}=\frac{u_{2, x}^{3}+3 u_{3, x}^{2}-3 u_{1, x} u_{2, x} u_{3, x}}{u_{3, x}^{3}} .
$$

Then the generalized Riemann equation at $N=3$ has the conserved density

$$
F=u_{3, x} G\left(\frac{u_{2, x}^{2}-2 u_{1, x} u_{3, x}}{u_{3, x}^{2}}, \frac{u_{2, x}^{3}+3 u_{3, x}^{2}-3 u_{1, x} u_{2, x} u_{3, x}}{u_{3, x}^{3}}\right)
$$

such that $\partial_{t}(F)=\partial_{x}\left(-u_{1} F\right)$.

Example 4.2. For the generalized Riemann equation at $N=4$, the fundamental dynamical variables are

$$
\alpha_{1}^{(1)}=Q_{3}, \quad \alpha_{2}^{(1)}=Q_{3}^{2}-2 Q_{2} Q_{4}, \quad \alpha_{3}^{(1)}=Q_{3}^{3}-3 Q_{2} Q_{3}+3 Q_{1}, \quad \alpha_{4}^{(1)}=2 Q_{4} Q_{0}-2 Q_{1} Q_{3}+Q_{2}^{2} .
$$

Taking $Q_{0}=1 / u_{4, x}$ and $Q_{i}=u_{i, x} / u_{4, x}(i=1,2,3,4)$ into account, we obtain

$$
\begin{aligned}
& \alpha_{1}^{(1)}=\frac{u_{3, x}}{u_{4, x}}, \quad \alpha_{2}^{(1)}=\frac{u_{3, x}^{2}-2 u_{2, x} u_{4, x}}{u_{4, x}^{2}}, \\
& \alpha_{3}^{(1)}=\frac{u_{3, x}^{3}-3 u_{2, x} u_{3, x} u_{4, x}+3 u_{1, x} u_{4, x}^{2}}{u_{4, x}^{3}}, \quad \alpha_{4}^{(1)}=\frac{u_{2, x}^{2}-2 u_{1, x} u_{3, x}+2 u_{4, x}}{u_{4, x}^{2}} .
\end{aligned}
$$

Hence, the quantity

$$
F=u_{4, x} G\left(\frac{u_{3, x}^{2}-2 u_{2, x} u_{4, x}}{u_{4, x}^{2}}, \frac{u_{3, x}^{3}-3 u_{2, x} u_{3, x} u_{4, x}+3 u_{1, x} u_{4, x}^{2}}{u_{4, x}^{3}}, \frac{u_{2, x}^{2}-2 u_{1, x} u_{3, x}+2 u_{4, x}}{u_{4, x}^{2}}\right)
$$

is conserved, and satisfies $\partial_{t}(F)=\partial_{x}\left(-u_{1} F\right)$ when the generalized Riemann equation at $N=4$ holds.

Two comments are in order. The conservation laws found by Olver and Nutku [9] for the Riemann equation (1.1) could be recovered from above general formulas by taking $N=1$. Also, we remark that, while the generalized Riemann equation at $N-1$ may be obtained from the equation at $N$, it does not mean that we may construct the conserved densities of the former from those of the latter. The obstacle is indeed the iterative relations (4.3), where $u_{N, x}$ appears as a denominator. 


\section{Conclusion}

The conserved densities, involving arbitrary smooth functions, have been worked out explicitly for the generalized Riemann equations, and in this way the previous results about this topic have been extended. As illustrated by the $N=2$ case, the proper changes of variables $\mathscr{T}_{1}$ and $\mathscr{T}_{2}$, which linearize Eqs. (2.3a)-(2.3b), convert the assumed conservation law (2.2) to a first order PDE with separable variables and result in the conserved densities of the type (2.9). It is noticed that for the Gurevich-Zybin, Monge-Ampere and two-component Hunter-Saxton equations, the conservation laws involving arbitrary functions are established by reducing those in Proposition 2.1. However, rather than concrete expressions, a formula is given by Proposition 2.2 for the conserved quantities. Thus, the conservation laws for these three systems may not be recovered from this Proposition. It is also believed that the existence of the conserved densities depending on arbitrary functions have their roots in the linearizable property of the generalized Riemann equations. However, further investigations are needed to reveal this deep connections for the generic case. It will be interesting to consider potential applications of the conserved densities presented in this paper, such as constructing new solutions, exploiting new nonlinear phenomenon and so on.

\section{Acknowledgments}

We greatly appreciate the anonymous referees' constructive comments, which are very helpful to improve the paper. This work is supported by the National Natural Science Foundation of China (grant numbers: 11271366, 11331008 and 11505284) and the Fundamental Research Funds for Central Universities.

\section{References}

[1] M. Antonowicz and A.P. Fordy, Coupled KdV equations with multi-Hamiltonian structures, Physica D 28 (1987) 345-357.

[2] J.C. Brunelli and A. Das, On an integrable hierarchy derived from the isentropic gas dynamics, J. Math. Phys. 45 (2004) 2633-2645.

[3] J. Golenia, M.V. Pavlov, Z. Popowicz and A.K. Prykarpatsky, On a nonlocal Ostrovsky-Whitham type dynamical system, its Riemann type inhomogeneous regularizations and their integrability, SIGMA. 6 (2010) 002 (13 pages).

[4] A.V. Gurevich and K.P. Zybin, Nondissipative gravitational turbulence, Sov. Phys. JETP. 67 (1988) $1-12$.

[5] A.V. Gurevich and K.P. Zybin, Large-scale structure of the Universe. Analytic theory, Phys.-Usp. 38 (1995) 687-722 .

[6] J.K. Hunter and R. Saxton, Dynamics of director fields, SIAM J. Appl. Math. 51 (1991) 1498-1521.

[7] J.K. Hunter and Y. Zheng, On a completely integrable nonlinear hyperbolic variational equation, Physica D 79 (1994) 361-384.

[8] F. Magri, A simple model of the integrable Hamiltonian equation, J. Math. Phys. 19 (1978) 1156-1162.

[9] P.J. Olver and Y. Nutku, Hamiltonian structures for systems of hyperbolic conservation laws, J. Math. Phys. 29 (1988) 1610-1619.

[10] M.V. Pavlov, The Gurevich-Zybin system, J. Phys. A: Math. Gen. 38 (2005) 3823-3840.

[11] Z. Popowicz, The matrix Lax representation of the generalized Riemann equations and its conservation laws, Phys. Lett. A 375 (2011) 3268-3272.

[12] Z. Popowicz and A.K. Prykarpatsky, The non-polynomial conservation laws and integrability analysis of generalized Riemann type hydrodynamical equations, Nonlinearity. 23 (2010) 2517-2537.

[13] A.K. Prykarpatsky, O.D. Artemovych, Z. Popowicz and M.V. Pavlov, Differential-algebraic integrability analysis of the generalized Riemann type and Korteweg-de Vries hydrodynamical equations, $J$. 
Phys. A: Math. Theor. 43 (2010) 295205 (13 pages).

[14] S. Sakovich, On a Whitham-type equation, SIGMA. 5 (2009) 101 (7 pages).

[15] K. Tian and Q.P. Liu, Conservation laws and symmetries of Hunter-Saxton equation: revisited, Nonlinearity. 29 (2016) 737-755.

[16] G.B. Whitham, Linear and nonlinear waves (Wiley, New York, 1974). 